$$
\begin{array}{llrl}
F(1)=1 & F(5)=88 & F(9)=1,097,780,312 \\
F(2)=1 & F(6)=1,802 & F(10)=376,516,036,188 \\
F(3)=2 & F(7)=75,598 & \\
F(4)=9 & F(8)=6,421,599 &
\end{array}
$$

\title{
REFERENCES
}

1. W. Ackermann, Die Widerspruchsfreiheit de allgemeinen Mengenlehre, Math. Ann. 114 (1937), 305-315.

2. J. L. Kelley, General topology, Van Nostrand, New, York, 1955.

University of California, Davis

\section{A NOTE ON THE GREATEST CROSSNORM}

\section{A. F. RUSTON}

Schatten has shown [5, Lemma 2, p. 323; 6, Lemma 3.7, p. 55] that, if $\mathfrak{M}$ is a closed subspace of a Banach space $\mathfrak{B}$, and there is a projection of $\mathfrak{B}$ onto $\mathfrak{M}$ with bound unity, then the greatest crossnorm on the tensor product $\mathfrak{B} \odot \mathfrak{R}$ is an extension of the greatest crossnorm on $\mathfrak{M} \odot \mathfrak{N}$ for any Banach space $\mathfrak{N}$.

Now it is known that there is a projection with bound unity of the second conjugate $\mathfrak{B}^{* *}$ of a Banach space $\mathfrak{B}$ onto $\mathfrak{B}_{0}$ (the canonical image of $\mathfrak{B}$ in $\mathfrak{B}^{* *}$ ) for conjugate spaces $\mathfrak{B}$ and for some others [3, p. 580], though not for all Banach spaces (cf. [7]). For such spaces, then, the greatest crossnorm on $\mathfrak{B}^{* *} \odot \mathfrak{N}$ is an extension of the greatest crossnorm on $\mathfrak{B}_{0} \odot \mathfrak{R}$. The purpose of this note is to show that the restriction to such spaces is unnecessary. (N.B. $\mathscr{B}$ is sometimes embedded in $\mathfrak{B}^{* *}$ by identifying it with $\mathfrak{B}_{0}$.)

Theorem. Let $\mathfrak{B}$ and $\mathfrak{R}$ be any Banach spaces. Then the greatest crossnorm on $\mathfrak{B}^{* *} \odot \mathfrak{N}$ is an extension of the greatest crossnorm on $\mathfrak{B}_{0} \odot \mathfrak{N}$ (where $\mathfrak{B}_{0}$ is the canonical image of $\mathfrak{B}$ in $\mathfrak{B}^{* *}$ ).

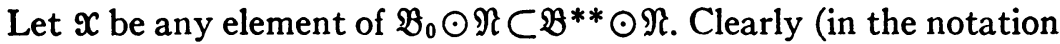
of $[2, \S 2.4$, pp. $347-351])$

$$
\gamma\left\{\mathfrak{B}^{* *} \odot \mathfrak{N}\right\}(\mathfrak{X}) \leqq \gamma\left\{\mathfrak{B}_{0} \odot \mathfrak{N}\right\}(\mathfrak{X})
$$

(since the infimum on the left-hand side is taken over a larger collection of expressions). On the other hand, there exists a continuous

Received by the editors October 5, 1961. 


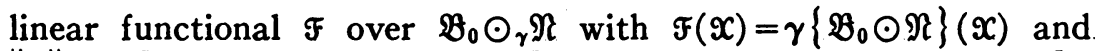
$\|\mathscr{F}\|=1[1$, Theorem 2.9 .3 , p. 19]. Now $\mathcal{F}$ can be associated (cf. [4, Theorem 1.2 , p. 78; 6, Theorem 3.2, p. 47]) with a continuous linear operator $T$ on $\mathfrak{R}$ into $\mathfrak{B}^{*}$ with the same norm as $\mathcal{F}$ by the rule

$$
\mathcal{F}(\tilde{x} \otimes y)=(T y)(x) \quad(x \in \mathfrak{B}, y \in \mathfrak{N}),
$$

where $\bar{x}$ is the canonical image of $x$ in $\mathfrak{B}^{* *}$. We now construct a continuous linear operator $T^{\prime}$ on $\mathfrak{N}$ into $\mathfrak{B}^{* * *}$ by defining $T^{\prime} y$ to be the canonical image of $T y$ in $\mathfrak{B}^{* * *}$ for each $y$ of $\mathfrak{R}$. This is associated with a continuous linear functional $\mathfrak{F}^{\prime}$ over $\mathfrak{B}^{* *} \odot_{\gamma} \mathfrak{N}$ with the same norm as $T^{\prime}$ by the rule

$$
\mathcal{F}^{\prime}(X \otimes y)=\left(T^{\prime} y\right)(X) \quad\left(X \in \mathfrak{B}^{* *}, y \in \mathfrak{N}\right)
$$

Then

$$
\mathcal{F}^{\prime}(\tilde{x} \otimes y)=\left(T^{\prime} y\right)(\tilde{x})=\tilde{x}(T y)=(T y)(x)=F(\tilde{x} \otimes y),
$$

and so $\mathcal{F}^{\prime}$ is an extension of $\mathcal{F}$, and

$$
\left\|F^{\prime}\right\|=\left\|T^{\prime}\right\|=\|T\|=\|F\|=1
$$

Hence

$$
\gamma\left\{\mathfrak{B}_{0} \odot \mathfrak{N}\right\}(\mathfrak{X})=|\mathfrak{F}(\mathfrak{X})|=\left|F^{\prime}(\mathfrak{X})\right| \leqq \gamma\left\{\mathfrak{B}^{* *} \odot \mathfrak{N}\right\}(\mathbb{X}) .
$$

This inequality, in conjunction with that above, shows that

$$
\gamma\left\{\mathfrak{B}^{* *} \odot \mathfrak{N}\right\}(\mathfrak{X})=\gamma\left\{\mathfrak{B}_{0} \odot \mathfrak{N}\right\}(\mathfrak{X}) .
$$

Since the element $\mathbb{X}$ of $\mathfrak{B}_{0} \odot \mathfrak{N}$ was arbitrary, this completes the proof of the theorem.

\section{REFERENCES}

1. E. Hille, Functional analysis and semi-groups, Amer. Math. Soc. Colloq. Publ. Vol. 31, Amer. Math. Soc., Providence, R. I., 1948.

2. A. F. Ruston, Direct products of Banach spaces and linear functional equations, Proc. London Math. Soc. 1 (1951), 327-384. 580.

3. - Conjugate Banach spaces, Proc. Cambridge Philos. Soc. 53 (1957), 576-

4. R. Schatten, The cross-space of linear transformations, Ann. of Math. (2) 47 (1946), 73-84.

5. - On projections with bound 1, Ann. of Math. (2) 48 (1947), 321-325.

6. - A theory of cross-spaces, Princeton Univ. Press, Princeton, N. J., 1950.

7. A. Sobczyk, Projection of the space $(m)$ on its subspace $\left(c_{0}\right)$, Bull. Amer. Math. Soc. 47 (1941), 938-947.

University of Sheffield, Sheffield, England 